\title{
LOS PROGRAMAS DE INVESTIGACION EN LAS UNIVERSIDADES COMO MEDIADORES DE UN PROCESO HACIA EL DESARROLLO DE ACTITUDES INVESTIGATIVAS FAVORABLES EN LOS FUTUROS PROFESIONALES
}

\author{
Manuel Erazo Parga \\ ProfesorDepartamento de Química \\ Universidad Pedagógica Nacional
}

\begin{abstract}
Resumen:Se trata de someter a juicio de profesores y estudiantes algunas consideraciones en relación con la investigación como una actividad propia de la razón de ser de la Universidad, de su importancia dentro del proceso de formación de los profesionales como punto de partida para el desarrollo del país en todos sus órdenes; partiendo de un análisis de la situación nacional se presentan algunos principios como propuesta a lo que debe ser ésta actividad en la Universidad Pedagógica en general y en la Facultad de Ciencia y Tecnología en particular, poniendo en consideración algunos elementos básicos necesarios para su organización, apoyo y gestión administrativa.
\end{abstract}

\section{En el contexto de la Universidad en general}

Uno de los tantos conceptos que con frecuencia son pronunciados por la mayor parte de las personas y cuya interpretación es más intuitiva que reflexiva, aún por algunos de sus propios integrantes, es el de Universidad; esto se relaciona con el hecho de que los procesos cotidianos, en el sentir de la mayor parte de la sociedad, no están mediados por la cultura y el saber, por lo que ha terminado asignándole el rol casi exclusivo de institución preparadora o capacitadora de recursos humanos para el empleo, una etapa más de estudios, después del bachillerato a la que hay que acceder, preferencialmente, con el fin de lograr alguna mediana preparación que posibilite el desarrollo de algunas competencias en los individuos para su vinculación al mercado laboral.

Esta pobre concepción de Universidad, y como punto de partida de éste análisis, debemos cambiarla por el de Universidad como una construcción intelectual; como una empresa de Hombres que labora por la recreación del pensamiento en la producción de nuevos conocimientos, y en este mismo sentido como un sector de la comunidad, cuyo objeto de trabajo es la Cultura en cuanto a su preservación, reelaboración, desarrollo y producción se refiere.

Si partimos de ésta concepción podemos observar entonces, que en Colombia nuestras universidades han centrado la mayor parte de sus esfuerzos a la preservación de la cultura de los oficios, trabajada casi con exclusividad en los currículos de formación de profesionales, y dedicando una parte muy pequeña, tanto del recurso humano como del debilitado presupuesto a la reelaboración, desarrollo y producción de nuevos patrimonios culturales.

Para corroborar lo anteriormente expuesto, podemos observar que la producción de nuevos conocimientos y tecnologías es hasta hoy una labor que muy tímidamente se pone de manifiesto en la Universidad colombiana, en donde la actividad intelectual entendida en su clímax de pureza sigue siendo algo exótico y que lo único que nos 
garantiza es seguir preparando "profesionales" para perpetuar el subdesarrollo y seguir manteniendo la imagen de país "exportador de materias primas", e "importador de tecnologías", muchas veces ya obsoletas en los países de origen y que a pesar de todo, cuando nos llegan, son subutilizadas.

En gran parte éste estado de cosas tiene su origen en el currículo escolar colombiano, en donde no se da prelación para que el alumno a través de los diferentes niveles de escolaridad, desarrolle y fomente la creatividad, el cuestionamiento, la reflexión y la explicación, propios del pensamiento critico-reflexivo que caracteriza al investigador; generalmente el sistema educativo fomenta a través del currículo escolar, el desarrollo del conocimiento enciclopédico, el cual se continúa propiciando en la Universidad; por tanto no se ésta fomentando la preparación de recursos humanos que lideren en nuestras universidades, de por si sin una alta tradición investigativa, la construcción de los paradigmas que fijen los derroteros del desarrollo científico y tecnológico del país, como producto de una investigación seria y sistemática de las realidades nacionales.

Para empezar la tan anhelada transformación de la educación para el desarrollo, el currículo escolar en general y el de la Universidad colombiana en particular, deben enrumbar sus acciones hacia la formación de profesionales con una mejor y mayor concepción investigativa, de tal manera que les permita ejercer su profesión enmarcados dentro de un quehacer permanentemente sistemático, apuntando hacia la transformación y desarrollo de la sociedad en todos sus órdenes.

El desarrollo de las sociedades en lo ideológico, artístico, científico y tecnológico solamente se ha dado gracias a la investigación sistemática, es decir al análisis reflexivo y crítico de la realidad, al constante estudio y cuestionamiento, al permanente estímulo y a la creatividad, en la búsqueda incesante de soluciones a los nuevos problemas que se presentan.

En la universidad colombiana, las tesis de grado constituyen hoy por hoy el único intento de fomentar la investigación a nivel de los pregrados, es así como en algunas universidades se han producido aportes investigativos de interés, sin embargo, en la mayoría, éste intento se ha quedado estancado, convirtiéndose en un agregado más de los planes de estudio y sólo como un requisito para obtener el título. En otras universidades ni siquiera se exige, o ha sido reemplazada por otro curso, mal llamado de postgrado, al final del cual se otorga el título.

La comunidad universitaria debe tomar conciencia de la importancia y de la necesidad de vincular la investigación al quehacer permanente del desarrollo académico como proceso para la formación de un buen grupo de profesionales investigadores en sus respectivas áreas de trabajo, los cuales tendrán la responsabilidad de conducir la sociedad colombiana hacia nuevos horizontes de desarrollo acorde con las necesidades futuras.

\section{En el contexto de la Universidad Pedagógica Nacional}

A la Universidad Pedagógica Nacional, le corresponde y como producto de un agotamiento explicativo al interior de sus estamentos básicos, establecer los criterios sobre lo que es y debe ser la educación en Colombia, trazar las políticas sobre desarrollo educativo y formación de docentes a todos los niveles y modalidades, de conformidad con las necesidades y prioridades nacionales; esto sólo se consigue a través de un proceso 
sistemático en el que cada una de sus unidades académicas estén conscientemente comprometidas en la identificación y solución de la problemática educativa del país.

Considero que las actividades investigativas que debe adelantar la Universidad dentro de los principios de una "Escuela de altos estudios en educación", fundamentalmente deben estar orientados a la producción de conocimiento pedagógico y didáctico en relación con los nuevos paradigmas que orienten la enseñanza y el aprendizaje de los diferentes saberes; es la comunidad de la Universidad Pedagógica Nacional y no otra, la que debe decir al país cuales son hoy en día las metodologías de enseñanza y aprendizaje de los saberes más eficaces y eficientes en concordancia con lo que al respecto se esta haciendo en el mundo, ésta es una responsabilidad eminentemente de la Universidad Pedagógica; producir conocimiento nuevo en las diferentes disciplinas no se descarta, pero es importante tener bien claro que ésta no es la función primordial de la Universidad Pedagógica, pues otras universidades tienen esta función y poseen una infraestructura con el escenario propio para la producción de conocimiento científico, tecnológico, artístico, etc.

Le corresponde a los profesores de cada una de las unidades académicas básicas, construir los problemas de investigación sobre los cuales se debe proyectar la investigación en la Universidad, dado que son ellos los que viven los problemas educativos actuales y quienes tienen la concepción más clara y real del proceso educativo colombiano, lo que les faculta para abordar trabajos relacionados con la identificación de los problemas y por ende proponer sus posibles soluciones.

La investigación en cada una de las unidades académicas, debe estar organizada a partir de equipos de investigadores con programas de investigación fruto del equipo, preferiblemente a largo plazo y con una financiación determinada; los programas de investigación como tales deben considerar además del objeto de conocimiento a investigar el espacio académico donde los futuros profesores de este país puedan lograr una formación investigativa de cierta rigurosidad, por tanto, a nivel de pregrado, son espacios que deben dar cierta relevancia al "proceso investigativo" sobre el "objeto a investigar".

Considero que la función principal de la investigación en los pregrados en general y en la Licenciatura en particular, debe estar orientada hacia la formación investigativa del futuro profesional, para que éste tenga la oportunidad de convertirse en un agente de cambio, con sentido innovador y crítico consciente de la problemática educativa de su país, y esto es más probable de adquirir si se está vinculado a un proceso serio y sistemático que le de la oportunidad de cambiar sus formas de pensamiento, readecuándolas a nuevas situaciones.

Aquí el "objeto a investigar", sin restarle la importancia que merece, se constituye en el vehículo en el que el futuro profesional hace su recorrido dentro de un proceso investigativo, donde su interés estará centrado más en el entorno, en el "paisaje" mismo, que en el vehículo en si.

Por tal razón pienso que el profesorado de cada una de las unidades académicas que se quiera comprometer con la investigación, debería ante todo conformar un equipo de trabajo alrededor d una determinada problemática con el fin de elaborar un programa de investigación, a mediano y largo plazo, para presentarlo en las diferentes instancias de la Universidad para su reconocimiento y búsqueda de apoyo en todos los órdenes. 
Esta puede ser una alternativa importante para que la investigación entre a ser reconocida como una actividad fundamental del docente que le garantice el apoyo suficiente, creando además la posibilidad muy importante, de generar grupos y equipos de investigadores, con lo cual se estaría dando un gran paso para preparar los recursos humanos necesarios para convertir a la Universidad en una verdadera asesora científica del Ministerio en Educación, ya que es muy difícil asesorar al Ministerio es aspectos educativos, científicos, innovadores y creativos, si éstos no son el fruto de los trabajos de investigación que los docentes de la Universidad hacen sobre la realidad educativa del país.

Se hace necesario entonces abrir el paso en el pregrado al estímulo, a la creatividad y crear las condiciones adecuadas para impulsar la investigación, introduciendo al alumno a través de un proceso formativo, que le de bases para un mejor desempeño profesional.

\subsection{La investigación en la Facultad de Ciencia y Tecnología.}

La investigación como ya se señaló, preferiblemente, debe estar soportada a partir de "Programas de investigación", los cuales serán formulados y liderados por equipos de investigadores, ojala de carácter interdisciplinario, en donde el equipo como tal se identificaría, entre otras cosas por:

1. Manejar una concepción general y clara acerca de los fines de la investigación dentro de los programas para la formación de profesores de ciencias.

2. Manejar una concepción de investigación dentro de los principios de rigurosidad que hoy manejan los investigadores.

3. Conocer un área temática relacionada con la enseñanza y/o el aprendizaje de las ciencias, en cuyos problemas de frontera se enmarca el problema del conocimiento.

4. Planear y organizar actividades investigativas, al interior de los programas, con una marcada tendencia a ofrecer espacios que faciliten vivenciar procesos formativos hacia la investigación en la acción educativa.

Los equipos se diferenciarán unos de otros, por el objeto de conocimiento alrededor del cual gira la problemática investigativa; hoy más que nunca estoy plenamente convencido de que el problema a investigar, con prelación, debe ser el planteado desde un referente didáctico, epistemológico y psicológico en relación con la enseñanza y/o aprendizaje de los conceptos científicos en general y los conceptos propios de cada una de las ciencias en particular, ya que, es sobre éstos aspectos sobre los cuales tenemos ante la comunidad educativa del país la responsabilidad de proponer alternativas de innovación pedagógica y didáctica.

Igualmente pienso que la investigación debe ser significativamente ambiciosa y debe contar con los recursos económicos para su desarrollo esto sólo se consigue cuando cada equipo plasme estas ideas en un documento "Programa de investigación del equipo" preferiblemente a mediano plazo, 2 a 4 años y lo someta a estudio para su financiación y donde entre otros se resalten:

1. Los principios filosóficos que inspiran las actividades investigativas del equipo, en relación con las finalidades del programa y su efecto en la formación científica y pedagógica del futuro profesor de ciencias. 
2. Los criterios de investigación que se manejan, representados desde la formulación del problema que maneja el programa, hasta los alcances, posibles metas parciales y los criterios de control y evaluación, pasando lógicamente por los objetivos, justificaciones, referente teórico desde el cual se plantea, sistemas de hipótesis, variables e indicadores y el diseño del programa con los posibles proyectos y subproyectos que lo conformarían.

3. El marco teórico, desde la perspectiva epistemológica, psicológica y didáctica en relación con la enseñanza y el aprendizaje de los contenidos de la ciencia en general y/o los de las disciplinas científicas en particular, los cuales hacen el papel de eje de la investigación y determinan a la vez las hipótesis de trabajo, los sistemas de trabajo e indicadores, así como el método para abordar la solución del problema y las características de los diferentes instrumentos a construir.

4. Recursos técnicos (necesidades de equipos y materiales); humanos (investigadores y coinvestigadores); financieros (costos/hora investigadores por tiempo de duración del proyecto; compra de materiales y equipos; alquiler de equipos; horas de computador; mecanografía; pasantías, etc.).

5. Cronograma general de actividades a los que estarían sometidos durante el proceso los coinvestigadores como parte formativa dentro del desarrollo de su tesis.

Otro aspecto que considero de vital importancia, en relación con la investigación, es el reconocimiento por parte de las instancias académico-administrativas de la Universidad, de que ésta es una labor prioritaria dentro de las actividades académicas que debe desarrollar cada profesor que tenga las capacidades para ello y esté interesado, y que por consiguiente se le asigne un merecido presupuesto y su correspondiente acreditación en la hasta hoy mal llamada "carga académica".

Igualmente, se hace necesario que los investigadores integren una especie de consejo de investigadores, con el fin de que sean los responsables de la gestión de apoyo, administración, control y evaluación de la investigación a nivel de la facultad, con el fin de poder administrar y controlar el proceso, y poder establecer unas condiciones que motiven a cada uno de los investigadores a trabajar en torno a la calidad de la investigación, condiciones de calidad que deben ser fijadas por consenso entre los diferentes equipos de investigación y cuyo único fin sea el de establecer criterios desde los cuales se puedan evaluar los proyectos y hacer las observaciones constructivas que proporcionen elementos de juicio para su mejoramiento, y así crear las condiciones para un permanente debate académico en relación con la problemática de la investigación.

Finalmente considero que, si la Universidad tiene como meta apoyar la investigación desde las bases académicas que son a mi entender los pilares para implementar cualquier política al respecto, y quienes se vincularían de diferentes maneras a la actividad investigativa, debe hacer un re-planteamiento a las funciones del CIUP, con el fin de reestructurarlo y organizarlo según las nuevas necesidades. Creo que éste Centro debe estar integrado por docentes investigadores de la Universidad, trabajando en equipo con otros profesionales que a juicio de los equipos sean necesarios, los cuales estarían adscritos al Centro en actividades investigativas, de consultoría y de asesoría propias del rol de la Universidad. 


\subsection{La investigación y los postgrados.}

Como una madurez académica de los pregrados, fundamentalmente reflejada sobre la base del desarrollo investigativo, manifestado a través de la consolidación de grupos de investigadores, fortalecimiento de las disciplinas, de las líneas y programas de investigación que apunten hacia la producción de nuevos conocimientos en al campo de la pedagogía y de la didáctica de los saberes, deben surgir los currículos de postgrado, como espacios específicos para la profundización en las disciplinas y la formación de investigadores en el campo de la docencia de éstas.

Estos deben ser montados sobre la base de programas de investigación que requieran de una madurez y un ambiente superior, para abordar la construcción y solución de problemas de mayor calidad y envergadura, donde ya el objeto a investigar, sin descuidar el proceso, requiere ser tratado dentro de un espacio de mayor madurez investigativa, estos espacios están llamados a ser líderes del desarrollo investigativo en cada una de las unidades académicas.

No se justifica abrir programas de postgrado que no estén consolidados sobre la base de la evolución de una infraestructura curricular investigativa del correspondiente pregrado, donde las experiencias obtenidas por profesores y alumnos hayan generado un ambiente propicio para institucionalizar la investigación dentro de los cánones de mayor rigurosidad a través de los postgrados; los investigadores, grupos de investigación, equipos de investigadores se comienzan a gestar y consolidar dentro de actividades experimentales que animan la vida académica de los pregrados en sus procesos de consolidación y maduración.

De este proceso es que surgen las denominadas líneas de investigación que irían a servir de soporte curricular del correspondiente postgrado, en donde las diferentes actividades académicas, tanto metodológicas como de asignaturas, están propuestas en el currículo como espacios de complementación y apoyo al programa o programas de investigación propios de cada una de las líneas de investigación.

Lo ideal es que cada equipo de investigadores, bajo cuya responsabilidad estaría la coordinación curricular de ésta actividad en el postgrado, debe estar consolidado alrededor de una problemática investigativa, en donde la dinámica de sus actividades está orientada a construir un discurso consistente y coherente, tal que le permita comunicarse e intercambiar experiencias con otros investigadores pares, tanto nacionales como internacionales.

Igualmente debe estar identificado e interesado en resolver una serie de problemas relacionados con los múltiples aspectos educativos que aquejan a los diferentes sectores de la población escolar diseñando, experimentando y ofreciendo alternativas didácticas y curriculares para su solución.

\section{La investigación como proceso formativo.}

Antes de empezar a presentar algunas sugerencias relacionadas con los componentes esenciales del proceso investigativo y lo que puede ser un programa de investigación, construido con el objetivo de abrir espacios para vincular al futuro profesional en una vivencia investigativa consistente y comprometida, es necesario afrontar una serie de dilemas críticos que sin lugar a duda inciden en la planeación y organización del proceso investigativo. 
En términos sencillos un programa de ésta naturaleza requiere de una claridad y una decisión frente a las siguientes alternativas sobre la forma de organizar y planificar las experiencias investigativas:

1. ¿Introducimos al estudiante en un proyecto de investigación, permitiéndole un avance a su propio ritmo, suministrándole orientación académica y asesoría explícita en la metodología de la investigación, tan sólo cuando lo solicite o se estime necesario hacerlo?

2. ¿Empezamos por proporcionarle la orientación académica y asesoría posibles sobre la metodología y problema a investigar, para que después la ponga en práctica gradualmente con base en los casos reales de su propia investigación?

La primera estrategia presenta la ventaja de proporcionar criterios fuertes de relevancia; es menos probable que le proporcione al estudiante una enseñanza inadecuada, otorga además bastante autoridad a éste frente a la figura del director o asesor, ya que es él mismo quien posee la experiencia de primera mano acerca del problema y su contexto, además, que recaerá sobre él la responsabilidad de informar a los directores sobre los detalles, dificultades e inquietudes que se le presenten durante el proceso investigativo.

El director asume así un papel más receptivo que el directivo y le exigirá una puesta al día en experiencias semejantes a las planteadas por los problemas de investigación, dado que los conocimientos necesarios para orientar y asesorar no suelen estar escritos en los libros.

Este enfoque puede suscitar una considerable incertidumbre y ansiedad en el alumno investigador, aunque presenta la ventaja de acercar al estudiante a la experiencia de investigar $y$, consiguientemente, a los problemas que surgen en el proceso de investigación. Igualmente despierta una considerable motivación y afán de compromiso de quienes se sienten capaces de tratar los problemas con destreza y confianza (R. Walkcr, 1989)

La segunda estrategia presenta la ventaja de que al comenzar con un período de preparación, cl estudiante toma conciencia de las posibilidades y limitaciones de la investigación antes de enfrentarse a los problemas que ésta le ofrece, restringiendo sus esfuerzos a tareas manejables y relativamente poco ambiciosas que se puedan realizar en un tiempo prudente y con unos recursos disponibles.

Se trata entonces de un enfoque, que si bien es cierto es restrictivo y convergente, donde prácticamente no interviene en la construcción del problema a investigar porque éste se le entrega ya parcial o totalmente construido, también es cierto que proporciona objetivos nítidos, un currículo lineal y unos procedimientos de evaluación claros; el principal peligro reside en la tendencia a transformar los problemas educativos en meros problemas académicos o técnicos.

A la hora de decidir cual sería la estrategia más adecuada, es preciso aclarar que las dos estrategias no son en sí tan incompatibles y que otros factores se deben tener en cuenta, tales como la dedicación del estudiante, lugar donde se desarrolla la investigación, así como las expectativas previas, motivaciones, habilidades y capacidades para comprender al estudiante por parte del director. 


\subsection{A cerca de la identificación del problema de conocimiento.}

Ahora si, después de haber presentado una serie de consideraciones en relación con los diferentes propósitos y estrategias que inspirarían la orientación de la actividad investigativa en la Universidad, desde luego recalcando su importancia como proceso formativo en el desarrollo de la capacidad de cuestionamiento, reflexión y explicación del futuro profesional, se debe entrar a plantear el ¿qué investigar?.

Si entendemos la investigación como una indagación sistemática y autocrítica, como un proceso que se acomete para resolver un problema de conocimiento, en donde el problema de conocimiento es todo aquello que se desea conocer y aún no se conoce o que se desea verificar porque aún no se ha verificado acerca de la realidad, observamos, que el ¿qué investigar? esta relacionado con el problema de conocimiento.

El problema de conocimiento es, por decirlo así, la puerta de entrada al proceso investigativo y éste debe ser identificado también dentro de un proceso sistemático y serio, pues el ¿qué investigar? es una pregunta o sistema de preguntas que surgen dentro de un discurso como problema de frontera del conocimiento, y es desde aquí donde comienza el proceso de formación del investigador.

Es necesario tener en cuenta que los problemas de investigación no suelen presentarse bien definidos a partir de la práctica educativa, sino que tienen que elaborarse, reformularse y retocarse una y otra vez hasta que adopten una forma que los haga abordables para la investigación. Se presentan incluso casos en que el problema está ya formulado en términos de investigación y el diseño preparado, pero el problema "real" no aparece con claridad hasta que surgen nuevos datos y se efectúan las interpretaciones preliminares. En este sentido, la progresión puramente lógica del enfoque racional, que aparece descrito en numerosos textos sobre investigación, resulta inapropiada. (Walker, 1989)

Con mucha frecuencia se encuentran "investigaciones" realizadas, donde el objeto a investigar nació de una simple sospecha, de una corazonada o de un impulso emocional sin el más mínimo análisis si ese era realmente un problema de conocimiento, desconociendo aún todo el referente teórico que involucra el "ámbito del problema".

Cabe así señalar que los problemas están planteados desde la frontera del conocimiento, en lo científico, en lo didáctico, en lo filosófico, en lo ideológico, en lo pedagógico, en lo psicológico, etc., es por esto que el problema de conocimiento del qué investigar debe estar dado como producto de un agotamiento explicativo al cuestionar la realidad educativa en que vivimos, lógicamente desde un referente teórico previamente construido, ya sea a partir de técnicas e instrumentos, para hacerlo de una manera directa o ya sea a partir de referentes secundarios, donde el problema de conocimiento esté planteado de una manera seria y sistemática.

Sólo así tenemos la seguridad de poder identificar plenamente los sistemas de variables que lo caracterizan al igual que los indicadores de comportamiento de cada una de las variables relacionadas con el problema, lo cual nos permitirá delimitarlo o precisarlo si es el caso, ya sea en el tiempo, en el espacio, en lo sociológico, económico, ideológico, etc. 


\subsection{A cerca del método.}

Una vez establecido claramente el problema de conocimiento, cabe la pregunta: ¿Cómo investigarlo? es aquí donde el conocimiento del investigador sobre el referente teórico que apoya la investigación adquiere vigencia cognitiva, es desde allí donde el diseño experimental y las formas operativas de abordar el problema se construyen con sentido, pues de las diferentes posibilidades de abordarlo, se elegirá aquella o aquellas que sean coherentes con la formulación del problema.

Aquí se hace necesario establecer la metodología de trabajo, la cual debe guardar una estrecha correlación con el proceso investigativo propuesto y con el problema de investigación formulado. Esto, si bien es cierto de por sí no garantiza los resultados de la investigación, por lo menos disminuye las posibilidades de error y permite a la vez evaluar y retroalimentar cada uno de los pasos dados e identificar posibles errores cometidos.

Una decisión clave en cualquier investigación es la selección de métodos, es una decisión que hay que tomar desde las fases iniciales de la investigación, y que una vez tomada no es fácil de revocar, aun cuando pueda enriquecerse posteriormente añadiendo métodos suplementarios; en la práctica, la elección de métodos concretos puede distorsionar la propia existencia del problema e investigación, ya que el método puede ser incluso una parte intrínseca e inherente al problema, en lugar de permanecer ajeno o desconectado del mismo.

Dentro de éstos criterios es que hoy en día se plantea la consistencia del proceso investigativo, o lo que en otros términos se plantea como rigurosidad investigativa y un futuro profesional debe ser consecuente con ésta realidad y la mejor manera de estimular el desarrollo de éstas concepciones investigativas en el futuro profesional, es la de introducirlo en procesos investigativos que le permitan desarrollar actitudes favorables hacia la investigación.

Sólo así y si empezamos desde ya, a preparar un profesor que realice su enseñanza desde una perspectiva investigativa, se puede tener la esperanza de un futuro mejor para nuestras juventudes, pues estaríamos dejando la exclusiva labor educativa actual de preservar la cultura por la del desarrollo del hombre para la innovación y producción de unos patrimonios culturales que nos den una identificación propia dentro del contexto de las sociedades del mundo.

\section{Bibliografía}

Elliot J. La investigación-acción en educación. Morata, Madrid, 1990

Erazo M. La información y la educación en Colombia, un problema real del cual hay que crear conciencia para obtener soluciones prácticas. Clepsidra No. 2, 1987, Universidad Pedagógica Nacional.

Erazo M. Consideraciones críticas para la aproximación de un modelo de enseñanzaaprendizaje de las ciencias por investigación. Investigación en la escuela, No. 12, 1990, Universidad de Sevilla, España.

Erazo M. ¿Para qué la investigación en el pregrado?. Departamento de Química, Universidad Pedagógica Nacional, Mimeógrafo, 1985.

Latorre A. El maestro investigador. Grao, Barcelona, 1987.

Stenhouse La investigación como base de la enseñanza. Morata, Madrid, 1987

Walker R. Métodos de investigación para el profesorado. Morata, Madrid, 1989. 